\title{
20 Jahre forschende Komplementärmedizin: Der Weg von der Avantgarde zum Mainstream
}

\author{
Gustav J. Dobos
}

Vorstand der Deutschen Gesellschaft für Naturheilkunde e.V., Kliniken Essen Mitte, Medizinische Fakultät, Universität Duisburg-Essen, Deutschland

1993 war ein Jahr der Gegensätze: Während in Jugoslawien im Kampf um die Souveränität der ethnischen Gruppen die Massaker des Bürgerkriegs anhielten und die Tschechoslowakei aufhörte zu existieren, trat im Herbst der Vertrag von Maastricht in Kraft und vollendete die europäische Einigung, an der seit den 1950er Jahren gearbeitet worden war. Auch die deutsche Einigung setzte ein Zeichen - mit neuen Postleitzahlen.

Vielleicht hilft es, sich das damalige Umfeld ins Gedächtnis zu rufen, um zu verstehen, was in den vergangenen 20 Jahren passiert ist. Denn die enormen Veränderungen in diesem Zeitraum sind das Thema dieser Ausgabe der Forschenden KomPLEMENTÄRMEDIZIN, die 2013 ihr 20-jähriges Bestehen feiert.

Das Jahr 1993 war auch für die Komplementärmedizin ein Wendepunkt: David Eisenberg und sein Team von der Harvard-Universität veröffentlichten ihren legendären Report «Unconventional Medicine in the United States» im New England Journal of Medicine und wiesen darin nach, dass bereits jeder dritte US-Bürger Methoden jenseits der Schulmedizin anwendete, um gesund zu werden. «Wir müssen uns also fragen, was letztlich den Gesundheitszustand unserer Nation beeinflusst - das, was die Medizin tut oder das, was die Medizin nicht tut?» war die ketzerische Frage, die David Eisenberg damals an die Öffentlichkeit stellte [1]. Die Medizin musste schmerzhaft feststellen, dass viele Menschen in Parallelwelten wechselten, weil ihnen offensichtlich Entscheidendes fehlte. Was in der Medizin falsch laufe, fragte sich auch der kanadische Mediziner David Sackett, der zu jener Zeit die Notwendigkeit einer «evidenzbasierten Medizin» postulierte. Wissenschaftliches Vorgehen sollte künftig bei Behandlungen eine zentrale Rolle spielen und das mehr oder weniger «eminenzbasierte» Handeln, das sich stärker auf die ärztlichen Hierarchien als auf nachprüfbares Wissen berief, ersetzen [2]. Eminenzen - oder, wenn man es freundlicher formuliert, große Vorbilder - spielten auch in der Komplementärmedizin eine große Rolle. Persönlichkeiten wie Paracelsus, Sebastian
Kneipp, Christoph W. Hufeland, Samuel Hahnemann, Rudolf Steiner und Maximilian O. Bircher-Benner waren der Anlass zur Gründung verschiedener Schulen der Naturheilkunde, die mehr oder weniger isoliert von- oder nebeneinander arbeiteten. Für den Laien waren Gemeinsamkeiten und Unterschiede kaum zu erkennen, auch nicht die Unterschiede zwischen Seriosität und Betrug, mit dem sich viel Geld machen ließ, was immer mehr selbsternannte Heiler mit kruden Theorien auf den Plan rief. In den USA hatte eine ähnlich unübersichtliche Heiler-Landschaft dazu geführt, dass im Rahmen der National Institutes of Health (NIH) ein Office of Alternative Medicine (OAM) gegründet wurde, das «unkonventionelle medizinische Praktiken» untersuchen und evaluieren sollte. Nach einigem Hin und Her und viel Kritik an der mangelhaften Unabhängigkeit des OAM von Lobbyisten wurde es 1998 reformiert und in das seither renommierte und wissenschaftlich anerkannte National Center for Complementary and Alternative Medicine (NCCAM) umgewandelt. Über 100 Millionen USD werden dort jährlich für die Erforschung von integrativen Heilverfahren ausgegeben; eine ähnlich große Summe investiert das National Cancer Institute in die Komplementärmedizin im Rahmen der Onkologie. Das NCCAM ist auch internationaler Vorreiter für die «Integrative Medizin» und überprüft wissenschaftlich komplementäre Heilverfahren mit dem Ziel, sie zum anerkannten Teil der medizinischen Behandlung zu machen.

Integration, nicht Abgrenzung, ist das Ziel, dem sich auch die Forschende KomplementärmedizIn verschrieben hat. Die naturwissenschaftlich orientierte Schulmedizin braucht diese Annäherung der Schul- und Komplementärmedizin dringend, denn rund 70\% der Krankheitskosten in entwickelten Staaten wie Deutschland entstehen durch chronische Leiden, die mit Naturheilverfahren oft effektiver behandelt werden können als mit den konventionellen Methoden. Die konsequente Suche nach Evidenz führt nicht nur in der Komplementärme-

\section{KARGER \\ Fax +497614520714 \\ Information@Karger.com}

www.karger.com (c) 2013 S. Karger GmbH, Freiburg

$1661-4119 / 13 / 0201-0010 \$ 38.00 / 0$

Accessible online at:

www.karger.com/fok
Prof. Dr. med. Gustav J. Dobos

Deutsche Gesellschaft für Naturheilkunde e.V.

Kliniken Essen-Mitte, Knappschafts-Krankenhaus

Am Deimelsberg 34a, 45276 Essen, Deutschland 
dizin zu einer Entmystifizierung. Die Evidenz (Grad I) onkologischer Leitlinien ist gering und liegt in Abhängigkeit von der Entität zwischen 0 und 20\% [3]. Die Versorgung mit einem Stent bei stabiler Angina pectoris zeigt keinerlei Vorteil im Vergleich zu einer rein medikamentösen Therapie bei deutlich erhöhtem Risiko [4]. Wiederholt konnte gezeigt werden, dass bei einer Kniegelenksarthrose weder die arthroskopische Lavage noch das operative Debridement eine über den Placebo-Effekt hinausgehende Wirkung hat [5]. Bestätigt werden die Ergebnisse durch aktuelle Cochrane-Metaanalysen $[6,7]$. Evidenzbasiert unwirksam und dennoch ein Spitzenreiter in der klinischen Praxis - welch riesengroßes Einsparungspotential!

Die Naturheilkunde/Komplementärmedizin hat ihre Stärken: mit Ansätzen, die nicht nur auf Symptome abzielen, sondern auf den gesamten Menschen mit seiner Konstitution, in der Suche nach Therapieansätzen mit weniger Nebenwirkungen und mit Impulsen, die die Selbstheilungskräfte anregen. Deshalb war es so wichtig, dass sich traditionelle Heilverfahren - wozu neben der europäischen Naturheilkunde auch die chinesische und die indische Medizin zählen - zunehmend der wissenschaftlichen Überprüfung stellen. Die Fördermittel zur wissenschaftlichen Erforschung sind hart umkämpft, denn im Gegensatz zur sogenannten Schulmedizin, für deren Forschung die Pharmaindustrie ein wesentlicher Geldgeber ist, fehlt bei den traditionellen Heilverfahren die finanzielle Unterstützung durch Drittmittel. Ohne die Hilfe privater Stiftungen wären die notwendigen Studien und die mittlerweile 8 Universitätsprofessuren in Deutschland undenkbar, da im Gegensatz zu den USA die komplementärmedizinische Forschung in Deutschland leider keinerlei staatliche Unterstützung erhält. Das erste und einzige Vorhaben, das von der Deutschen Forschungsgemeinschaft (DFG) unterstützt wird, ist eine Studie der Berliner Universitätsklinik Charité zu Heuschnupfen und Akupunktur. Dennoch bewegt sich etwas: z.B. das paneuropäische Forschungsnetz CAMbrella, gefördert mit 1,5 Millionen EUR durch die Europäische Union, in dem 16 Wissenschaftseinrichtungen aus 12 Partnerländern gemeinsam an einer «Roadmap» für komplementärmedizinische Forschung arbeiten [8]. Zudem gibt es das Kompetenznetz Komplementärmedizin in der Onkologie, das mit Mitteln der Deutschen Krebshilfe untersucht, welche ergänzenden oder komplementären Heilverfahren es in der Krebsmedizin gibt, und wie Patienten selbst ihre Behandlung sinnvoll unterstützen können. Die Deutsche Krebshilfe fördert das Projekt über einen Zeitraum von 3 Jahren mit 2,5 Millionen EUR. Heute scheint gerade die Integrative Onkologie ein großes Potenzial zu besitzen, Naturheilkunde mit Mind-Body-Medizin in der Regelversorgung umzusetzen [9]. Das Interesse von onkologischer Seite ist groß und eine Reihe von Zentren in Deutschland arbeitet bereits an der Umsetzung. Prognosen kündigen an, dass in 10 Jahren, ähnlich wie in den USA, jedes Comprehensive Cancer Center in Deutschland die Integrative Onkologie anbieten wird, um seinen Patienten auch Therapien zur Reduktion von Nebenwirkungen der Krebsbehandlung, zur Stärkung der Selbstheilungskräfte und zur Besserung der Lebensqualität anzubieten. Jetzt geht es darum, die notwendigen Standards dafür zu setzen. 2 komplementäre Therapieansätze, die nach der bisherigen Datenlage besonders vielversprechend sind, sollen hier nicht unerwähnt bleiben, da sie das Potenzial zu besitzen scheinen, nicht nur die Nebenwirkungen $\mathrm{zu}$ verringern und die Lebensqualität zu steigern, sondern ebenfalls die Prognose der Erkrankten zu verbessern. Zum einen handelt es sich dabei um Mind-Body-Medizin-Programme im Rahmen der Ordnungstherapie bei Prostatakarzinom, zum anderen um das Fasten als begleitende Therapie während der Chemotherapie. Nach Abschluss weiterer kontrollierter Studien wären diese gut geeignet, als reguläre Therapieoptionen in die Regelversorgung integriert zu werden. Eine jüngst im New England Journal of Medicine veröffentlichte Studie beispielsweise zeigte an 700 Patienten mit lokalisiertem Prostatakarzinom, dass es für ihr Überleben keinerlei Rolle spielte, ob sie sich operieren ließen oder nicht. Wohl aber war die Lebensqualität der Operierten aufgrund von Problemen der Inkontinenz und des Potenzverlustes deutlich schlechter [10]. «Wait and see» scheint beim lokalisierten Prostatakarzinom nach den vorliegenden Befunden eine gleichwertige Behandlungsoption zu sein. Sobald sich die Ergebnisse dieser Studie in der Bevölkerung entsprechend verbreitet haben, wird das Interesse an der Wait-and-see-Option rasant ansteigen. Das wirft die Frage auf, was der Patient sinnvollerweise in der Wartezeit machen kann, um seine Prognose zu verbessern.

Dean Ornish zeigte in seinem «Prostate Cancer Lifestyle Trial», wie ein Mind-Body-Medizin-Programm, bestehend aus Lebensstilmodifikationen mit veganer Diät, Bewegungstraining $(6 \times 30$ min Walking pro Woche $)$ und Stressbewältigung (Yoga, Atemtechniken und Meditation), den PSA-Wert reduzieren kann. Auch nach 1-2 Jahren war die Anzahl der notwendigen konventionellen Eingriffe wie Prostataektomie und Strahlen- oder Hormonbehandlung signifikant reduziert [11]. Offensichtlich zeigen die ordnungstherapeutischen Verfahren auch auf Gen-Expressionsebene eine deutlich positive Wirkung [12]. Interessant ist in diesem Zusammenhang auch die Tatsache, dass die Leitlinien zur Behandlung des Prostatakarzinoms einen Evidenzgrad besitzen, der nur zu $4 \%$ auf positiven Metaanalysen aus randomisierten kontrollierten Studien (RCTs) basiert. Lebensstilinterventionen in Sinne Ornishs sind also auch vonseiten einer evidenzbasierten Therapieoption eine sinnvolle Behandlung für Patienten, die bereit sind, aktiv zu werden - und wie wir aus den USA wissen, waren an Prostata erkrankte Männer ursprünglich unter den ersten Patienten, die komplementärmedizinische Verfahren in einem Maße nachfragten, das die Fachgesellschaften zwang, umzudenken. Die Mind-Body-Medizin kann in vielen Fällen einem Vergleich mit den konventionellen Verfahren standhalten [13].

Erste Studien weisen darauf hin, dass Fasten die Nebenwirkungen der Chemotherapie deutlich mildern kann. Das zeigte 
zum Beispiel die Arbeitsgruppe um Valter D. Longo von der University of Southern California. Allein 2 Fastentage vor und nach der Gabe von Chemotherapeutika verbesserten nicht nur das subjektive Befinden, sondern auch den Behandlungserfolg: Die Krebszellen reagierten empfindlicher auf die Chemotherapie, und die Nebenwirkung der Chemotherapie wurde deutlich abgemildert, ohne dass die Wirksamkeit auf den Krebs sich verringerte $[14,15]$. Erste vielversprechende Studien sind abgeschlossen; jetzt geht es darum, größere RCTs durchzuführen, um indikations- und diagnosespezifische Fasten-Strategien zu entwickeln. Der Nutzen der Komplementärmedizin lässt sich inzwischen in immer mehr Studien nachweisen - auch dank Fachzeitschriften wie der ForsCHENDEN KomplementÄrmedizin. Doch nun müssen diese Ergebnisse auch noch ihren Weg durch die Institutionen machen.

Was hat sich bei den Ärzten in den vergangenen 20 Jahren getan? Inzwischen ist eine junge Generation von Ärzten nachgerückt, die gegen die hierarchischen Strukturen und die Art der Patientenbehandlung aufbegehrt, die unser Gesundheitssystem kennzeichnen. Sie denkt intensiv über Defizite in der Medizin nach, mangelhafte Kommunikation, die fehlende Integration biopsychosozialer Faktoren, den drohenden Burnout im Beruf und die Wichtigkeit von Selbstfürsorge - auch für den Arzt. Die jungen Ärzte bringen ein Denken mit, das ganzheitlich orientiert und deshalb auch für vieles in der Komplementärmedizin aufgeschlossen ist [16]. Die Voraussetzung für die Kompatibilität mit der und die Integration in die konventionelle Medizin ist aber auch in diesem Fall der Nachweis von Evidenz. Darin unterscheidet sich die junge Ärztegeneration von ihren teilweise schwärmerischen Vorgängern. Der nächste Schritt ist deshalb das Einbringen komplementär- und integrativmedizinischen Wissens in die Leitlinien der Fachgesellschaf- ten - ein Ziel das die Deutsche Gesellschaft für Naturheilkunde (DGNHK) verfolgt, deren Organ die ForschendE KoMPLEMENTÄRMEDIZIN seit 2007 ist. Die Zeitschrift ist in MEDLINE gelistet und besitzt seit Jahren einen im deutschsprachigen Raum beachtlichen Impact Factor.

An dieser Stelle möchte ich der Forschenden KomplemenTÄRMEDIZIN zu ihrem 20-jährigen Jubiläum ganz herzlich gratulieren, den Mitarbeitern für ihre sorgfältige Arbeit danken und viel Freude und Erfolg bei der zukünftigen Arbeit wünschen!

Die Wochenzeitung Die Zeit stellt in einer aktuellen Titelgeschichte zum Thema «Patienten sind wichtiger als Profit» ein Manifest auf und fordert eine menschliche Medizin. Das klingt zunächst wie ein frommer, jedoch in der Regel unerhörter Wunsch. Aber etwas könnte diesmal anders sein! Neben dem zunehmenden ökonomischen Druck auf das Gesundheitssystem, nicht zuletzt aufgrund einer durch Lobbyisten geprägten Politik, lässt der immer häufiger ausbleibende Nachweis einer Evidenz konventioneller Therapieansätze bei hohen Kosten und einer gleichzeitigen Zunahme der Evidenznachweise innerhalb der Complementary and Alternative Medicine (CAM) das konventionelle System wanken und schafft Raum für Neues. «Medizin und Menschlichkeit» ist auch das Thema verschiedener, mittlerweile internationaler Studentengruppierungen der neuen Generation der Ärzte von Morgen. Diese Ärzte sind zunehmend weiblich. Die rapide Zunahme des Anteils von Ärztinnen, verbunden mit einem wachsenden Ärztemangel, wird die Krankenhauslandschaft verändern. Geschäftsführungen und ärztliche Leitungen werden herausgefordert sein, Strukturen an diese Entwicklung anzupassen. Möglicherweise werden infolgedessen sogar alte Hierarchien in der Medizin aufweichen. Die Medizin wird weiblicher. Ich bin sehr gespannt darauf!

\section{Literatur}

1 Eisenberg DM, Kessler RC, Foster C, Norlock FE, Calkins DR, Delbanco TL: Unconventional medicine in the United States. Prevalence, costs, and patterns of use. N Engl J Med 1993;328:246-252.

2 Sackett DL, Rosenberg WM, Gray JA, Haynes RB, Richardson WS: Evidence based medicine: what it is and what it isn't. BMJ 1996;312:71-72.

3 Poonacha TK, Go RS: Level of scientific evidence underlying recommendations arising from the $\mathrm{Na}$ tional Comprehensive Cancer Network clinical practice guidelines. J Clin Oncol 2011;29:186-191.

4 Stergiopoulos K, Brown DL: Initial coronary stent implantation with medical therapy vs medical therapy alone for stable coronary artery disease: metaanalysis of randomized controlled trials. Arch Intern Med 2012;172:312-319.

5 Moseley JB, O'Malley K, Petersen NJ, Menke TJ, Brody BA, Kuykendall DH, Hollingsworth JC, Ashton CM, Wray NP: A controlled trial of arthroscopic surgery for osteoarthritis of the knee. N Engl J Med 2002;347:81-88.

6 Laupattarakasem W, Laopaiboon M, Laupattarakasem P, Sumananont C: Arthroscopic debridement for knee osteoarthritis. Cochrane Database Syst Rev 2008;23:CD005118.
Reichenbach S, Rutjes AW, Nüesch E, Trelle S, Jüni P: Joint lavage for osteoarthritis of the knee. Cochrane Database Syst Rev 2010;12:CD007320.

8 www.cambrella.eu/home.php (Zugriff vom 06.02. 2013).

-9 Dobos GJ, Voiss P, Schwidde J, Choi KE, Paul A, Kirschbaum B, Saha FJ, Kuemmel S: Integrative oncology for breast cancer patients: introduction of an export-based model. BMC Cancer 2012;12:539.

10 Wilt TJ, Brawer MK, Jones KM, Barry MJ, Aronson WJ, Fox S, Gingrich JR, Wei JT, Gilhooly P, Grob BM, Nsouli I, Iyer P, Cartagena R, Snider G, Roehrborn C, Sharifi R, Blank W, Pandya P, Andriole GL, Culkin D, Wheeler T; Prostate Cancer Intervention versus Observation Trial (PIVOT) Study Group: Radical prostatectomy versus observation for localized prostate cancer. New Engl J Med 2012;367:203-213.

11 Ornish D, Weidner G, Fair WR, Marlin R, Pettengill EB, Raisin CJ, Dunn-Emke S, Crutchfield L, Jacobs FN, Barnard RJ, Aronson WJ, McCormac P, McKnight DJ, Fein JD, Dnistrian AM, Weinstein J, Ngo TH, Mendell NR, Carroll PR: Intensive lifestyle changes may affect the progression of prostate cancer. J Urol 2005;174:1065-1070.
12 Ornish D, Magbanua MJ, Weidner G, Weinberg V, Kemp C, Green C, Mattie MD, Marlin R, Simko J, Shinohara K, Haqq CM, Carroll PR: Changes in prostate gene expression in men undergoing an intensive nutrition and lifestyle intervention. Proc Natl Acad Sci USA 2008;105:8369-8374.

13 Dobos G, Paul A: Mind-Body-Medizin: Die moderne Ordnungstherapie in Theorie und Praxis. München, Elsevier, 2011

14 Raffaghello L, Lee C, Safdie FM, Wei M, Madia F, Bianchi G, Longo VD: Starvation-dependent differential stress resistance protects normal but not cancer cells against high-dose chemotherapy. Proc Natl Acad Sci USA 2008;105:8215-8220.

15 Safdie FM, Dorff T, Quinn D, Fontana L, Wei M, Lee C, Cohen P, Longo VD: Fasting and cancer treatment in humans: a case series report. Aging (Albany NY) 2009;1:988-1007.

16 www.faz.net/aktuell/feuilleton/forschung-und-lehre/ generation-y-der-aufstand-der-jungen-aerzte11783044.htm (Zugriff vom 06.02.2013). 